ARTICLE

Received 14 Dec 2014 | Accepted 26 Mar 2015 | Published 8 May 2015 | Updated 17 Jun 2015

DOI: $10.1038 /$ ncomms 8062

OPEN

\title{
Ventral hippocampal afferents to the nucleus accumbens regulate susceptibility to depression
}

Rosemary C. Bagot ${ }^{1}$, Eric M. Parise ${ }^{2, \star}$, Catherine J. Peña ${ }^{1, \star}$, Hong-Xing Zhang ${ }^{3}$, lan Maze ${ }^{1,3}$, Dipesh Chaudhury ${ }^{3}$, Brianna Persaud ${ }^{1}$, Roger Cachope ${ }^{4}$, Carlos A. Bolaños-Guzmán ${ }^{2}$, Joseph F. Cheer ${ }^{4}$, Karl Deisseroth ${ }^{5}$, Ming-Hu $\operatorname{Han}^{1,3}$ \& Eric J. Nestler ${ }^{1}$

Enhanced glutamatergic transmission in the nucleus accumbens (NAc), a region critical for reward and motivation, has been implicated in the pathophysiology of depression; however, the afferent source of this increased glutamate tone is not known. The NAc receives glutamatergic inputs from the medial prefrontal cortex (mPFC), ventral hippocampus (vHIP) and basolateral amygdala (AMY). Here, we demonstrate that glutamatergic VHIP afferents to NAc regulate susceptibility to chronic social defeat stress (CSDS). We observe reduced activity in vHIP in mice resilient to CSDS. Furthermore, attenuation of vHIP-NAc transmission by optogenetic induction of long-term depression is pro-resilient, whereas acute enhancement of this input is pro-susceptible. This effect is specific to VHIP afferents to the $N A c$, as optogenetic stimulation of either mPFC or AMY afferents to the NAc is pro-resilient. These data indicate that $\mathrm{VHIP}$ afferents to NAc uniquely regulate susceptibility to CSDS, highlighting an important, novel circuit-specific mechanism in depression.

\footnotetext{
${ }^{1}$ Fishberg Department of Neuroscience, Icahn School of Medicine at Mount Sinai, New York, New York 10029, USA. ${ }^{2}$ Department of Psychology and Program in Neuroscience, Florida State University, Tallahassee, Florida 32306, USA. ${ }^{3}$ Department of Pharmacology and Systems Therapeutics, Icahn School of Medicine at Mount Sinai, New York, New York 10029, USA. ${ }^{4}$ Department of Anatomy and Neurobiology, University of Maryland School of Medicine, Baltimore, Maryland, 21201, USA. ${ }^{5}$ Departments of Bioengineering and Psychiatry and Behavioral Sciences, Stanford University, Stanford, California 94305 , USA. * These authors contributed equally to this work.. Correspondence and requests for materials should be addressed to E.J.N. (eric.nestler@mssm.edu).
} 
A lterations in nucleus accumbens (NAc), a region critical for reward and motivation, are implicated in the pathophysiology of depression ${ }^{1-7}$. Glutamatergic transmission in medial NAc is increased in mice exhibiting depression-like behaviour after chronic stress ${ }^{3-5}$. However, the role of presynaptic changes in driving stress-induced postsynaptic adaptations in NAc medium spiny neurons (MSNs) is not well understood. The NAc integrates cortico-limbic afferents with dopaminergic modulation from the ventral tegmental area ${ }^{8,9}$. Integration occurs at the cellular level, with individual NAc MSNs receiving convergent glutamatergic projections from ventral hippocampus (vHIP), medial prefrontal cortex (mPFC) and basolateral amygdala (AMY), in addition to other regions such as the thalamus ${ }^{8,10-13}$. Complex competitive interactions between inputs gate postsynaptic MSN responses to afferent activation to direct the finely tuned integration of executive control from mPFC, conditioned associations and emotion from AMY and contextual, spatial and emotion-related inputs from vHIP to orchestrate adaptive motivated behaviour $8,9,14,15$.

mPFC activity is reduced in depressive-like states in rodents, and optogenetic activation of mPFC, a manipulation that, among other actions, increases glutamate release in NAc, is antidepressant ${ }^{16-18}$. These findings are paradoxical in view of observed increases in mini EPSC frequency ${ }^{4}$ and AMPA current ${ }^{3}$ in NAc MSNs of stress-susceptible mice, which point to stress-induced facilitation of both pre- and postsynaptic mechanisms of glutamate transmission. This raises the question of whether chronic stress may produce pathway-specific alterations, with distinct glutamatergic inputs exerting opposing effects on depression-like behaviour ${ }^{19-22}$. To examine this possibility, we investigated regulation of vHIP-NAc, mPFC-NAc and AMYNAc projections by chronic social defeat stress (CSDS), a validated mouse model of depression ${ }^{1,2}$. Examining immediate early gene (IEG) expression as an indicator of neuronal activity and electrophysiological correlates of presynaptic neurotransmitter release, we report afferent-specific CSDSinduced adaptations in vHIP and mPFC. Using optogenetic manipulations to bidirectionally control afferent-specific synaptic function, we demonstrate a unique role for vHIP-NAc in driving depression-like behavioural phenotypes.

\section{Results}

CSDS oppositely regulates activity of vHIP and mPFC. We examined mRNA expression of two IEGs, Arc and Egr1, in mPFC, vHIP and AMY of susceptible and resilient mice versus stress-naive controls to assess basal activation of these brain regions $48 \mathrm{~h}$ after CSDS. Mice were determined to be susceptible or resilient based on their social interaction ratio (time in interaction zone with social target/time with target absent) in a social interaction test $24 \mathrm{~h}$ after CSDS (susceptible ratio $<1$; resilient ratio $>1$; Supplementary Fig. 1). This designation has been highly validated in previous studies and shown to correlate with other depression-related, but not anxiety-related, behavioural abnormalities ${ }^{1-3}$. Both Arc and Egr1 transcript levels were reduced in vHIP of resilient mice versus controls, whereas $A r c$, but not Egr1, was decreased in $\mathrm{mPFC}$ of susceptible mice and neither transcript was altered in AMY (Fig. 1a-f). To identify vHIP, mPFC and AMY neurons projecting to NAc, we injected the retrograding AAV2/5-CaMKIIa-EYFP virus into NAc before CSDS. Double-immunofluorescence labelling revealed increased EGR1-positive EYFP-labelled vHIP neurons in susceptible mice (Fig. 1g). No significant effects were observed in NAc-projecting $\mathrm{mPFC}$ and AMY neurons (Fig. 1i,k). ARC protein levels are too low under these conditions to perform the equivalent analysis. Reduced IEG expression in these regions of resilient versus susceptible mice suggests that decreased vHIP activity might be pro-resilient, whereas decreased mPFC activity is pro-susceptible.

To assess afferent-specific glutamatergic synaptic transmission in NAc, mice were injected with (non-retrograding) AAV5CaMKIIa-ChR2-EYFP virus in vHIP, mPFC or AMY (Supplementary Fig. 2). The CaMKIIa promoter targets viral infection to glutamatergic neurons within the injected brain regions (see Methods), and we confirmed that optically induced currents are blocked completely by glutamate receptor antagonists (Supplementary Fig. 2c). Six weeks later, when robust transgene expression was seen in nerve terminals in NAc, mice were exposed to CSDS. Using whole-cell patch-clamp in brain slices, optogenetically stimulated paired-pulse responses were recorded in medial NAc from control, susceptible and resilient mice beginning $48 \mathrm{~h}$ after CSDS. The resulting ratio of excitatory postsynaptic current (EPSC) amplitudes to paired stimulations is indicative of vesicle release probabilities and suggestive of changes in glutamate release ${ }^{23}$. Paired-pulse ratios (PPRs) of vHIP afferent-stimulated responses in NAc were increased in resilient relative to susceptible mice, suggesting a potential decreased probability of glutamate release (Fig. $1 \mathrm{~m}$ ). In contrast, PPRs of $\mathrm{mPFC}$ afferent-stimulated responses were decreased in resilient animals relative to susceptible mice (Fig. 10). PPRs of AMY afferent-stimulated responses were not regulated by CSDS (Fig. 1q). These findings suggest that glutamate release at vHIPNAc synapses may be decreased in resilient mice, whereas mPFCNAc synaptic glutamate release is increased in resilient mice.

LTD attenuation of vHIP-NAc synaptic transmission is proresilient. To determine whether decreasing glutamatergic synaptic transmission at vHIP-NAc synapses could promote resilience in previously defeated mice, we injected AAV5CaMKIIa-ChR2-EYFP or AAV5-CaMKIIa-EYFP into vHIP and 6 weeks later exposed mice to CSDS. Optic fibres targeting medial NAc were then surgically implanted, and optogenetic low frequency stimulation (LFS; $473 \mathrm{~nm}, 10 \mathrm{~min}, 1 \mathrm{~Hz}, 4 \mathrm{~ms}$ pulse width) of ChR2-infected terminals in NAc was used to induce long-term depression (LTD) of MSN EPSCs. One week after surgery, we delivered LFS to reduce vHIP-NAc synaptic transmission $45 \mathrm{~min}$ before social interaction testing (Fig. 2a); LTD-like responses were electrophysiologically validated by optogenetic terminal stimulation of inputs in brain slices and in vivo (Fig 2b-d; Supplementary Fig. 3a-c) as previously described $^{24}$. vHIP ChR2-infected mice spent more time interacting with a target mouse in comparison with EYFP-infected controls (Fig. 2e) and similar to what is normally observed in resilient mice indicating a pro-resilient effect. The same manipulations did not affect social interaction in mice injected with AAV5CaMKIIa-ChR2-EYFP in mPFC or AMY (Fig. 2f,g). vHIP, mPFC or AMY injected stress-naive controls were unaffected by optical stimulations (Supplementary Fig. 3).

Enhancement of vHIP-NAc synaptic transmission is pro-susceptible. To examine whether conversely increasing glutamate synaptic transmission in NAc could increase stress susceptibility, vHIP terminals in medial NAc were acutely stimulated to increase glutamate release at vHIP-NAc synapses during social interaction testing following CSDS (Fig. 3a; fidelity of acutely evoked EPSCs was validated in brain slices Fig. 3b-d; and in vivo, Supplementary Fig. $4 \mathrm{a}-\mathrm{c})$. Mice receiving acute optical stimulation $(4 \mathrm{~Hz}, 5 \mathrm{~ms})$ during social interaction testing spent less time interacting with a target animal and increased time in corner zones compared with non-stimulated controls (Fig. 3e,h). To assess whether increasing glutamate release selectively at 


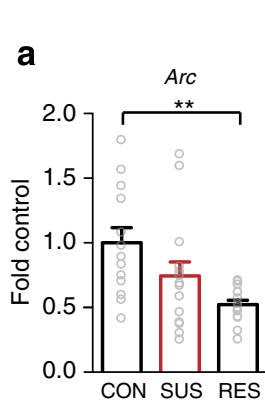

vHIP

b
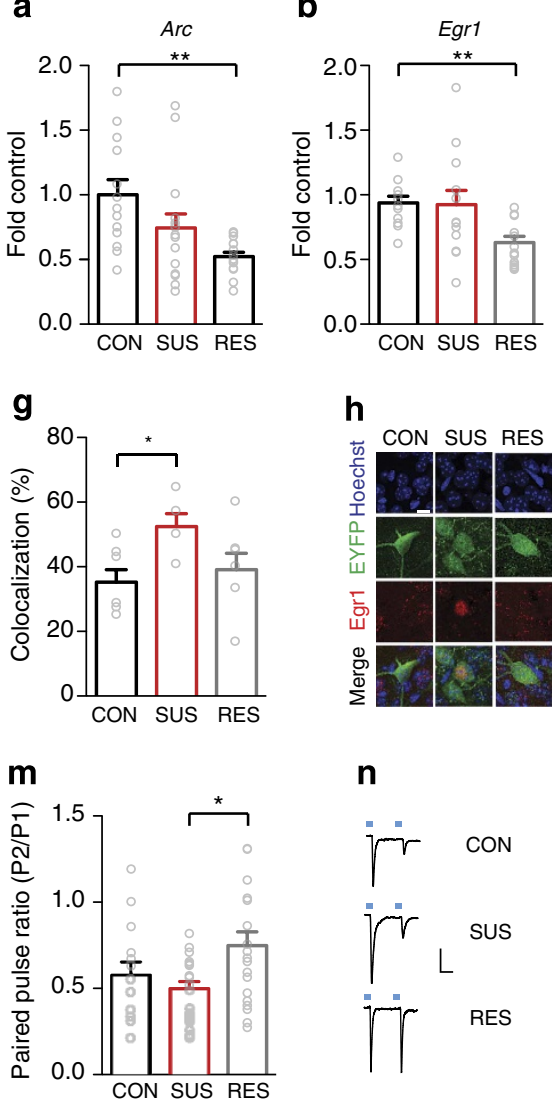

h

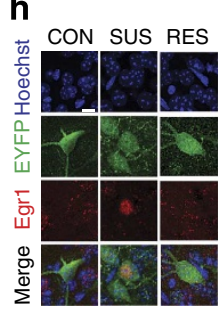

n

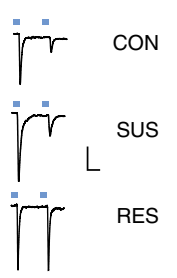

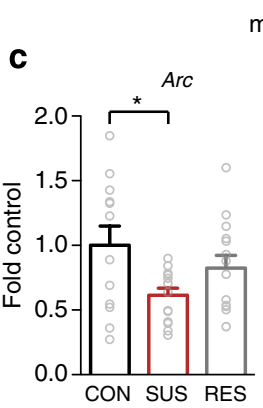
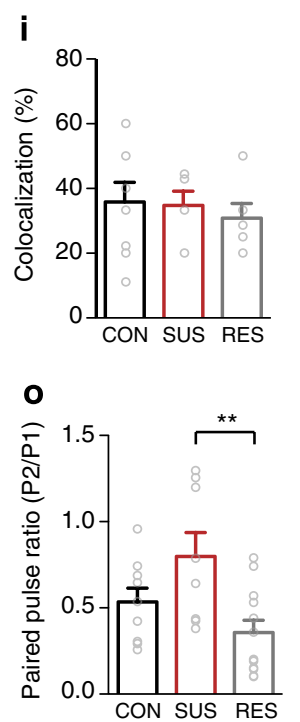
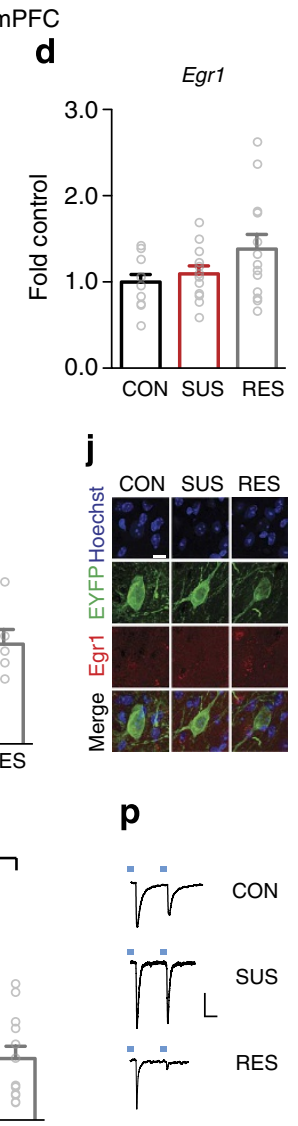
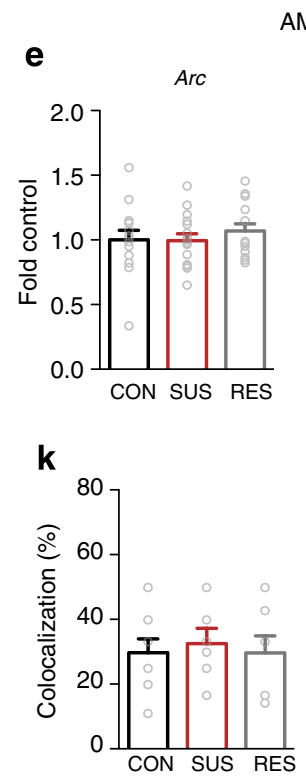

AMY

$f$
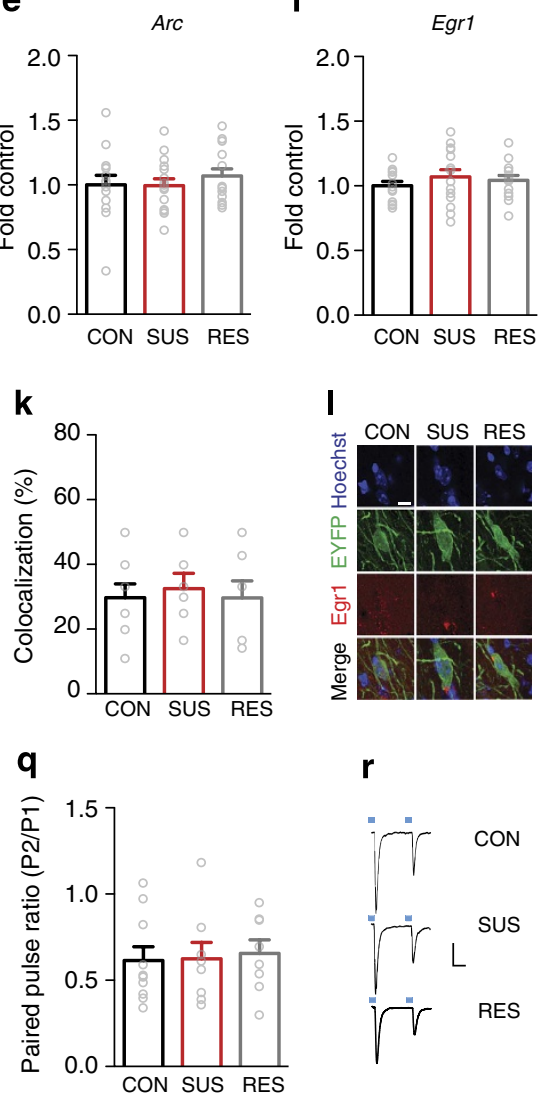

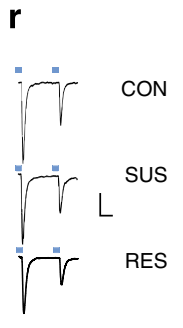

Figure 1 | Opposing effects of CSDS on vHIP and mPFC IEG expression and synaptic function. mRNA expression of two immediate early genes in vHIP of control (CON), susceptible (SUS) and resilient (RES) mice. (a) Arc $\left(F_{2,40}=6.485, P<0.01\right.$, post hoc $\left.{ }^{\star} P<0.01, n=13,15,15\right)$ and $(\mathbf{b})$ Egr1 $\left(F_{2,36}=5.415, P<0.01\right.$, post hoc $\left.{ }^{\star \star} P<0.01, n=12,14,13\right)$ were decreased in vHIP of RES mice $48 \mathrm{~h}$ post-defeat. In contrast, (c) Arc expression was decreased in mPFC of SUS mice $\left(F_{2,37}=3.431, P<0.05\right.$, post hoc CON versus SUS $\left.{ }^{*} P<0.05, n=12,14,14\right)$. (d) Egr1 expression was not significantly different $(n=11,13,12)$. Neither Arc (e) nor Egr1 (f) expression was regulated by defeat in AMY $(n=14,15,15)$. A greater percentage of EYFP retrogradely labelled NAc-projecting neurons were EGR1 positive in VHIP $(\mathbf{g})$ of SUS mice compared to controls $\left(F_{2,16}=3.618, P<0.05\right.$, post hoc $\left.{ }^{\star} P<0.05, n=7,5,7\right)$. EGR1 levels in $\mathrm{MPFC}(\mathbf{i})$ and AMY (k) in NAc-projecting neurons were not significantly regulated by defeat. Representative images show Hoecsht nuclear stain (blue) in EYFP labelled EGR1 (red) staining in NAc-projecting neurons in VHIP (h), mPFC (j) and AMY (I) labelled by retrograde AAV2/5-CaMKIla-EYFP injected into NAc (scale bar $10 \mu \mathrm{m}$ ). In RES relative to SUS mice, paired-pulse ratios $\left(\mathrm{P}_{2} / \mathrm{P}_{1} ; 100 \mathrm{~ms}\right.$ interpulse interval) of optically evoked EPSCs in NAc shell MSNs were increased from $(\mathbf{m})$ vHIP $\left(F_{2,50}=4.023, P<0.05\right.$, post hoc ${ }^{\star} P<0.05, n=14,20,19$ cells from $n=6,7,6$ mice $)$ and decreased from (o) mPFC

$\left(F_{2,26}=5.459, P<0.01\right.$, post hoc ${ }^{\star \star} P<0.01, n=9,8,12$ cells from $n=5,4,7$ mice). Paired-pulse ratios from AMY ( $q$ ) were not regulated by defeat $(n=10,8,8$ cells from $n=4,3,4$ mice). Representative EPSCs evoked by optical stimulation of vHIP (n), mPFC (p) and AMY (r) ChR2-expressing terminals. Vertical scale bar (n) 80 pA, (p,r) 40 pA, horizontal scale bar 40 ms. Blue squares indicate light pulse delivery. One-way-ANOVAs with Bonferroni post hoc tests. Grubb's test was used to detect and remove statistical outliers in panels a-f. Error bars represent s.e.m.

mPFC-NAc synapses could increase resilience to stress, similar to the effect of global mPFC stimulation ${ }^{16}$, we acutely stimulated mPFC terminals in medial NAc. Mice receiving acute stimulation of mPFC terminals in NAc spent more time interacting with a target mouse and less time in the corner zones (Fig. 3f,i). Acutely stimulated mice that were injected intra-AMY with AAV5-CAMKIIa-ChR2-EYFP spent more time interacting with a target mouse (Fig. 3g,j), similar to the effect of mPFC stimulation. As an additional control, we confirmed that light stimulation of EYFP-injected control mice and non-stimulated ChR2-injected mice exhibited equivalent behavioural responses (Supplementary Fig. 5)

Acute stimulation of each of the three distinct NAc afferents, vHIP, mPFC or AMY, did not affect anxiety-like behaviour in an open field test, indicating that the effects on social interaction are not confounded by effects on anxiety (Supplementary Fig. 4d-f). Moreover, acute vHIP terminal stimulation increased immobility in the forced swim test, confirming a pro-depressant behavioural effect (Supplementary Fig. 4g). In contrast, acute AMY terminal stimulation decreased immobility, whereas mPFC terminal stimulation had no effect (Supplementary Fig. 4h,i). Stimulation of each of the three afferents increased locomotor activity in the open field test (Supplementary Fig. 4j-1). Effects of acute stimulation on social-avoidance behaviour require previous stress exposure: manipulations in stress-naive-mice did not alter social interaction. Of note, AMY stimulation decreased exploration of the empty interaction zone, but time spent interacting with a target mouse was not affected (Supplementary Fig. 6).

\section{Discussion}

NAc MSNs receive dense glutamatergic projections from vHIP, mPFC and AMY, among other regions. Here, we present data suggesting that vHIP-NAc synaptic transmission is selectively increased in mice susceptible to CSDS, and demonstrate that in vivo optogenetic manipulations of vHIP-NAc synaptic 
a

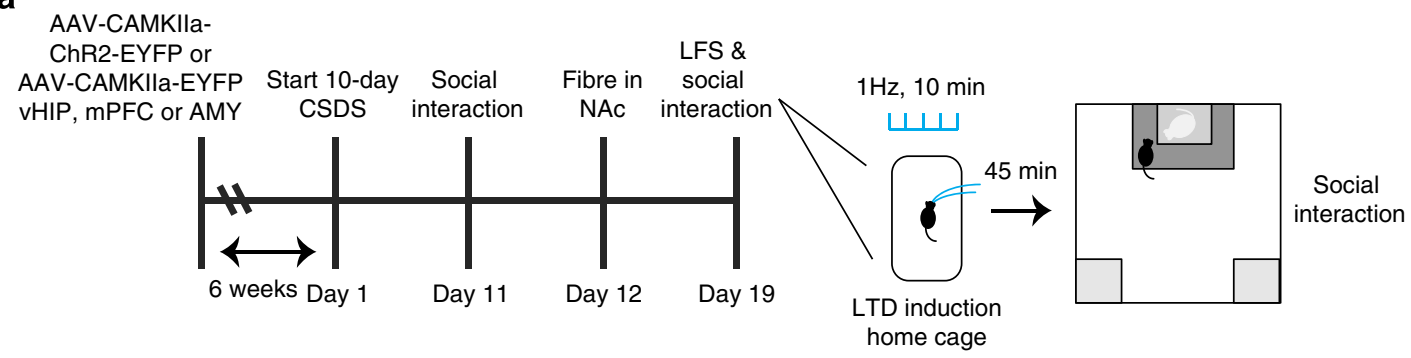

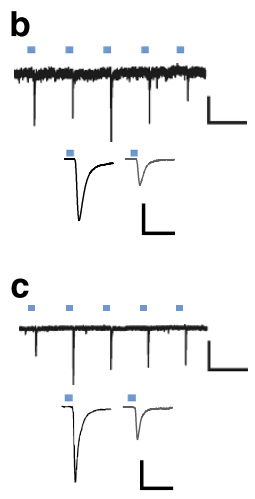

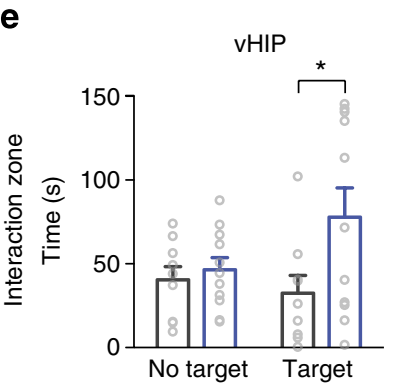

h

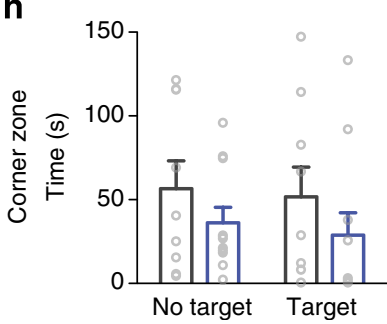

f

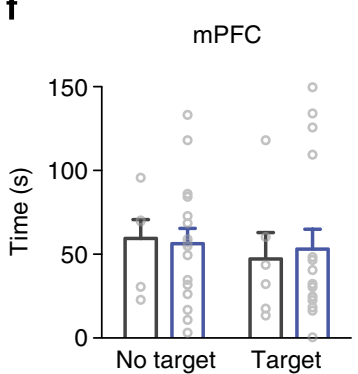

g

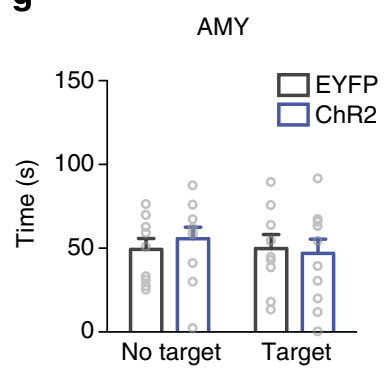

i

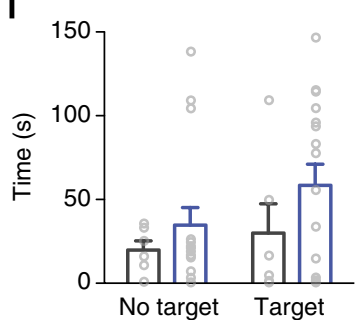

j

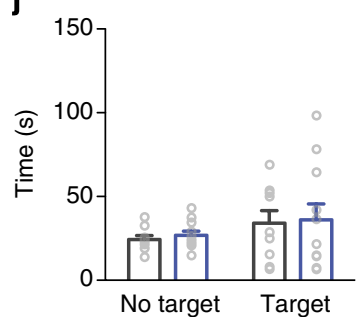

Figure 2 | LTD at vHIP-NAc induces resilience to CSDS. (a). Schematic of experimental time-course. Representative traces validating that $1 \mathrm{~Hz}$ stimulation of ChR2-expressing vHIP (b) (scale bar 200 pA, 1s), mPFC (c) (scale bar 50 pA, 1s) and AMY (d) (scale bar 200 pA, 1s) terminals reliably evoke EPSCs with temporal fidelity in NAc MSNs (upper panels). $10 \mathrm{~min}, 1 \mathrm{~Hz}$ stimulation (low frequency stimulation; LFS) induces LTD of evoked EPSCs measured $45 \mathrm{~min}$ after LFS (b-d, lower panels; scale bars 80 pA, $50 \mathrm{~ms}$ ) 45 min post-LFS (grey traces) compared to pre-LFS baselines (black traces). Blue squares indicate light pulse delivery. (e) In vivo, LFS of vHIP-NAc synapses in defeated mice increased time spent in the interaction zone in the presence of a target $\left(F_{1,18}=5.274\right.$ interaction effect, $P<0.05$, post hoc $\left.{ }^{\star} P<0.05, n=9,11\right)$ but did not alter time spent in corners (h). Stimulation of mPFC-NAc $(\mathbf{f} ; n=6$, 16) or AMY-NAc synapses $(\mathbf{g} ; n=9,11)$ had no effect on time spent in interaction or corner zones $(\mathbf{i}, \mathbf{j})$. Two-way repeated measures ANOVA with Bonferroni post hoc tests. 'EYFP' and 'ChR2' denote AAV5-CaMKIla-EYFP and AAV5-CaMKIla-ChR2-EYFP, respectively. 'No target' and 'Target' indicate absence or presence of a target mouse during testing. Error bars represent s.e.m.

transmission bidirectionally regulate depression-like behaviours. In contrast, our findings suggest that mPFC-NAc synaptic transmission is decreased in susceptible animals, and show that increasing either mPFC or AMY synaptic transmission by acute stimulation in vivo promotes resilience.

The mechanism of these distinct pathway-specific adaptations is unclear. Opposing effects of vHIP versus mPFC and AMY synaptic transmission could reflect the relative strengths of the examined inputs; vHIP projections to NAc shell are denser than those arising from mPFC or AMY ${ }^{12}$. Also, although all of these inputs are rewarding in stress-naive mice, stronger stimulations of $\mathrm{mPFC}$ and AMY are necessary to achieve this rewarding effect $^{12,25,26}$. Previous work has demonstrated that blockade of AMPA receptors in NAc increases resilience to stress 3 . This finding was difficult to integrate into a circuit-level understanding given: (1) established findings that activity of $\mathrm{mPFC}$, a major source of glutamatergic innervation in the NAc, is reduced in depression and (2) the therapeutic efficacy of mPFC stimulation ${ }^{16,27-30}$. The results of the present study suggest that the therapeutic effect of NAc AMPA blockade is mediated by vHIP-NAc glutamate synapses. In addition, it is possible that these afferents differentially target the two MSN subtypes (Drd1- versus Drd2-expressing) to mediate opposing behavioural effects ${ }^{26,31,32}$ and that stress may shift the cell-typespecific balance of connectivity in this circuit, a possibility that warrants direct examination.

The uniquely pro-depressant role of vHIP-NAc glutamate projections in chronically stressed mice opposes established roles for these projections in facilitating reward in stress-naive mice $12,25,26$. A recent paper examined the regulation of plasticity in the vHIP-NAc pathway in another rodent depression model (learned helpless rats) by electrically stimulating the fimbria and recording cell firing in the $\mathrm{NAc}^{33}$. Intriguingly, this study reported that a stimulation that produces long-term potentiation in control rats induced LTD in learned helpless rats. Synaptic plasticity at glutamatergic synapses is tightly regulated by homeostatic and metaplastic processes. Increased activity at glutamatergic synapses can facilitate the later induction of $\mathrm{LTD}^{34-36}$. Although methodological and species differences between the two studies limit the ability to draw direct comparisons, it is interesting to speculate whether this earlier finding might also suggest that glutamatergic synaptic transmission in the vHIP-NAc pathway is potentiated in learned helpless rats, thus allowing later electrical stimulation to induce 

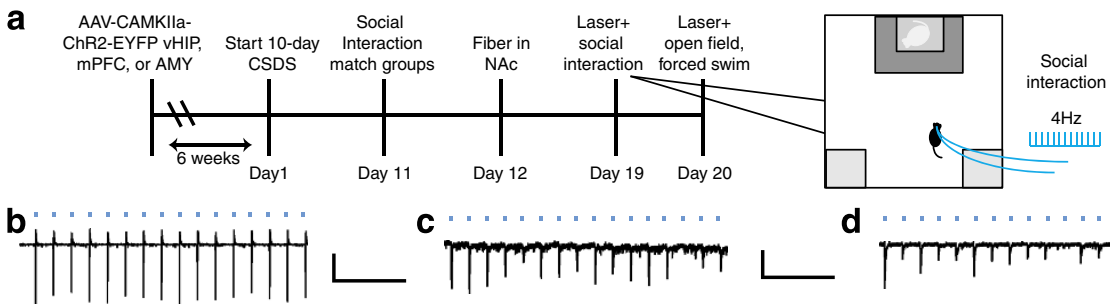

C

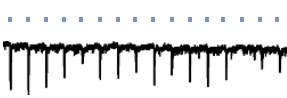

d
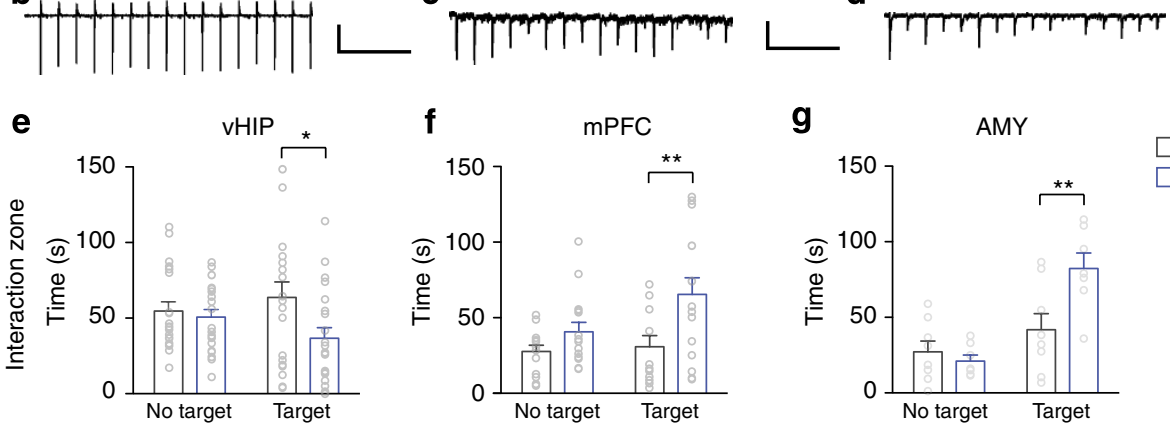

f

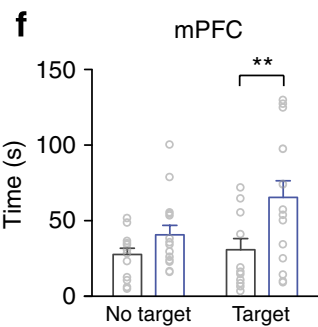

g
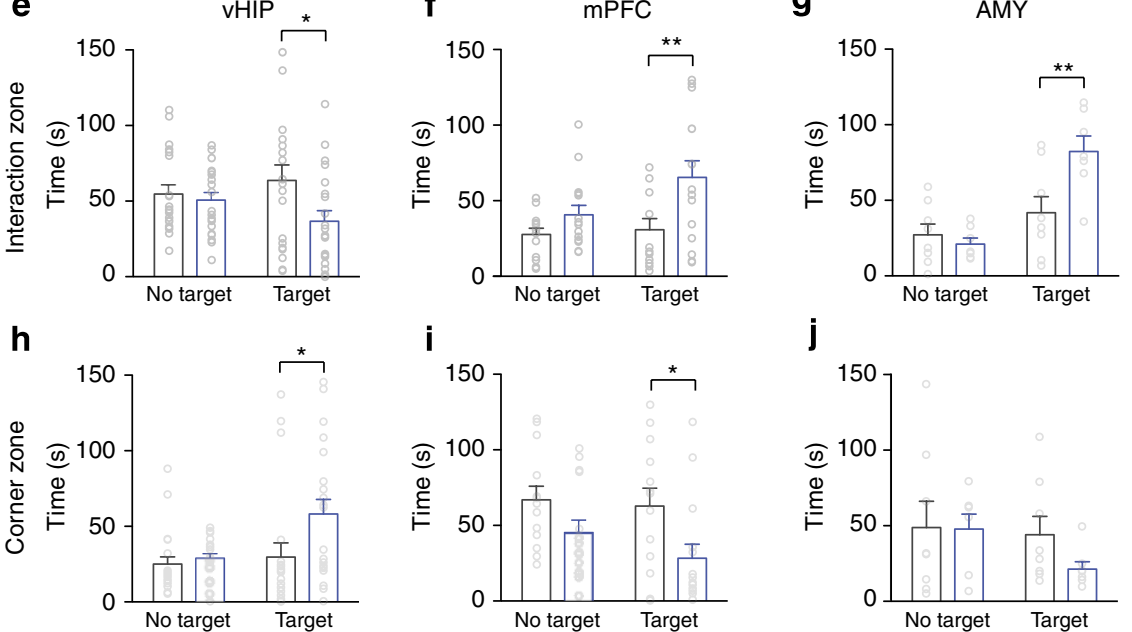

Figure 3 | Acute stimulation of vHIP versus mPFC or AMY terminals in NAc promotes opposite behavioral responses to CSDS. (a). Schematic of experimental time course. Representative traces validating that $4 \mathrm{~Hz}$ stimulation of vHIP (b) (scale bar $200 \mathrm{pA}, 1 \mathrm{~s}$ ), mPFC (c) (scale bar $50 \mathrm{pA}, 1 \mathrm{~s}$ ) and AMY (d) (scale bar 50 pA, 1s) terminals in NAc slices evokes EPSCs. Blue squares indicate light pulse delivery. In vivo, $4 \mathrm{~Hz}$ stimulation of vHIP terminals in NAc shell (e) reduced time spent interacting with a target mouse $\left(F_{1,39}=4.274\right.$ interaction effect, $P<0.05$, post hoc $\left.{ }^{\star} P<0.05, n=20,21\right)$ and $(\mathbf{h})$ increased time spent in corners $\left(F_{1,39}=6.560\right.$ target effect, $F_{1,39}=4.271$, stimulation effect, $P<0.05$, post hoc $\left.{ }^{*} P<0.05, n=20,21\right)$. Stimulation of $\mathrm{mPFC}$ terminals in NAc (f) increased time spent interacting with a target mouse $\left(F_{1,26}=9.174\right.$ stimulation effect, $P<0.01$, post hoc $\left.{ }^{\star \star} P<0.01, n=13,15\right)$ and $(\mathbf{i})$ decreased time spent in corners $\left(F_{1,26}=7.735, P<0.01\right.$, post hoc $\left.{ }^{\star} P<0.05, n=13,15\right)$. Stimulation of AMY axons in NAc (g) increased time spent interacting with a target mouse $\left(F_{1,14}=5.4284\right.$ interaction effect, $P<0.01$, post hoc $\left.{ }^{\star \star} P<0.01, n=8\right)$ but $(\mathbf{j})$ did not affect time spent in corners. Two-way repeated-measures ANOVAs with Bonferroni post hoc tests. 'No stim' and ' $4 \mathrm{~Hz}$ ' denote non-stimulated controls versus $4 \mathrm{~Hz}$ stimulated mice. 'No target' and 'Target' indicate absence or presence of a target mouse during testing. Error bars represent s.e.m.

LTD. While human structural imaging studies have reported decreased hippocampal volume in depressed subjects, functional imaging studies report indicators of increased hippocampal glutamate associated with familial risk of depression, increased hippocampal activation during negative information processing and an association between decreased hippocampal metabolism and antidepressant responses ${ }^{27,28,37,38}$. The mechanism of stressdependent regulation of vHIP glutamate projections may reflect an interaction with alterations in dopaminergic input to NAc. Ventral tegmental area dopamine neuron burst firing is increased in susceptible mice ${ }^{1,39,40}$, and phasic dopamine release in NAc has been shown to facilitate vHIP-NAc inputs ${ }^{14}$. Interestingly, vHIP-NAc stimulation may also increase VTA dopamine neuron population activity ${ }^{41}$

Our findings highlight a complex afferent-specific role for glutamatergic signalling in NAc in depression-associated behaviours. Chronic stress may shift the balance away from mPFC towards vHIP control, creating a reinforcing loop further enhancing vHIP-NAc synaptic transmission. Circuit-level therapeutic interventions that attenuate presynaptic vHIP overactivation in addition to targeting postsynaptic alterations in NAc may constitute more effective treatment strategies.

\section{Methods}

Experimental animals. Male 6-8 week-old C57BL/6 J mice, and 6-month-old CD1 retired breeders, were maintained on a 12-h light-dark cycle (lights on at 0700 hours) at $22-25^{\circ} \mathrm{C}$ with ad libitum access to food and water. C57 mice were housed
5 per cage except following defeat experiments and after fiber implantation surgery at which point mice were singly housed. All experiments were conducted in accordance with the guidelines of the Institutional Animal Care and Use Committee at Icahn School of Medicine at Mount Sinai. All behavioural testing occurred during the animals' light cycle. Experimenter was blinded to experimental group and order of testing was counterbalanced during behavioural experiments and across days for electrophysiology experiments. For in vivo LTD experiments (Fig. 2), animals were randomized within cages before surgery. For acute stimulation experiments (Fig. 3), groups were matched on post-defeat social interaction scores to generate two groups with equivalent means and variance.

RNA isolation and qPCR. Mice were killed $48 \mathrm{~h}$ after CSDS (see below), brains were removed, coronally sliced and mPFC, vHIP and AMY tissue was rapidly dissected and frozen on dry ice. RNA isolation, qPCR and data analyses were performed as described ${ }^{1}$. Briefly, RNA was isolated with TriZol reagent (Invitrogen) and purified with RNAeasy micro kits from Qiagen. All RNA samples were determined to have $260 / 280$ and $260 / 230$ values $\geq 1.8$. Reverse transcription was performed using iScript (BioRad). qPCR using SYBR green (Quanta) was carried out with an Applied Biosystems 7900HT RT-PCR system with the following cycle parameters: $2 \mathrm{~min}$ at $95^{\circ} \mathrm{C} ; 40$ cycles of $95^{\circ} \mathrm{C}$ for $15 \mathrm{~s}, 59^{\circ} \mathrm{C}$ for $30 \mathrm{~s}, 72^{\circ} \mathrm{C}$ for $33 \mathrm{~s}$; and graded heating to $95^{\circ} \mathrm{C}$ to generate dissociation curves for confirmation of single-PCR products. Data were analysed by comparing $\mathrm{C}(\mathrm{t})$ values of conditions tested (control versus susceptible or resilient mice) using the $\Delta \Delta \mathrm{C}(\mathrm{t})$ method $^{42}$. qPCR primers: Egrl FWD: $5^{\prime}$-GAGGAAGTTTGCCAGGA GTG-3', Egrl REV: 5'-GAGTAGGAAGTGGGCACAGG-3'; Arc FWD: 5'-GAAG TGGTGGGAGTTCAAGC-3', Arc REV: 5'-TCCTCAGCGTCCACATACAG-3'.

Immunohistochemistry. Forty-eight hours after CSDS (see below), mice were anaesthetized with chloral hydrate and transcardially perfused with $0.1 \mathrm{M}$ PBS followed by $4 \%$ paraformaldehyde in PBS. Brains were postfixed overnight and then cryopreserved in $30 \%$ sucrose. Brains were sectioned on a freezing microtome at $35 \mu \mathrm{m}$. Sections were rinsed three times in PBS and blocked in $4 \%$ normal 
donkey serum (NDS) with $0.5 \%$ Triton-X in PBS (PBST) for $2 \mathrm{~h}$ at room temperature. Sections were then incubated in primary antibodies overnight at $4{ }^{\circ} \mathrm{C}$ (chicken anti-GFP, \#AB13970, Abcam, 1:500 and rabbit anti-Egr1, \#4154 S, Cell Signaling, 1:1,000) in PBST. Sections were then washed three times in PBS and incubated with secondary antibodies (donkey anti-rabbit Cy3, donkey anti-chicken Alexa Fluor 488; 1:500, Jackson ImmunoResearch) for $2 \mathrm{~h}$ at room temperature. Sections were washed three times in PBS. Hoechst stain (Invitrogen) was added to the final wash for $25 \mathrm{~min}$. Sections were washed one final time before mounting and coverslipping with ProLong Gold (Invitrogen).

Stereotaxic surgery and viral vectors. Adenoassociated virus (AAV) vectors expressing channelrhodopsin-2 (ChR2) fused with enhanced yellow fluorescent protein (EYFP) under the control of the CaMKIIa promoter (AAV5-CaMKIIaChR2-EYFP) were used for optical activation of glutamatergic afferent terminals of NAc (mPFC, vHIP and AMY). CaMKIIa expression is specific to excitatory glutamatergic neurons ${ }^{43}$, and the use of this promoter in viral vectors has been shown to target such neurons ${ }^{12,44,45}$. AAV5-CaMKIIa-ChR2-EYFP and AAV5CaMKIIa-EYFP were purchased from the University of North Carolina Vector Core. To label neurons projecting to NAc, retrograding AAV2/5-CaMKIIa-EYFP purchased from University of Pennsylvania Viral Core was infused into NAc. (Note that AAV2/5 represents a different serotype than the AAV5 vector, even though the UPenn Core labels it as 'AAV5.') For stereotaxic surgeries, mice were anaesthetised with a mixture of ketamine $\left(100 \mathrm{mg} \mathrm{kg}^{-1}\right)$ and xylazine $\left(10 \mathrm{mg} \mathrm{kg}^{-1}\right)$ and positioned in a small-animal stereotaxic instrument (Kopf Instruments). The skull surface was exposed and 33-gauge syringe needles (Hamilton) were used to bilaterally infuse $0.5 \mu \mathrm{l}$ AAV5-CaMKIIa-ChR2-EYFP or control vector AAV5-CaMKIIa-EYFP at a rate of $0.1 \mu \mathrm{min}^{-1}$ into $\mathrm{mPFC}$ (bregma coordinates: anterior/posterior, $1.7 \mathrm{~mm}$; medial/lateral, $0.75 \mathrm{~mm}$; dorsal/ventral, $-2.5 \mathrm{~mm} ; 15^{\circ}$ angle; targeting infralimbic prefrontal cortex), vHIP (bregma coordinates: anterior/posterior, $-3.7 \mathrm{~mm}$; medial/lateral, $3 \mathrm{~mm}$; dorsal/ventral, $-4.8 \mathrm{~mm} ; 0^{\circ}$ angle; targeting ventral subciulum) or AMY (bregma coordinates: anterior/posterior, $-1.6 \mathrm{~mm}$; medial/lateral, $-3.1 \mathrm{~mm}$; dorsal/ventral, $-4.9 \mathrm{~mm} ; 0^{\circ}$ angle; targeting basolateral amygdala). The targeted regions all project to the NAc shell region targeted by our in vivo and in vitro manipulations ${ }^{12}$. Chronically implantable optic fibers constructed with $200 \mu \mathrm{m}$ core 0.22 NA optic fiber (Thor Labs) threaded through ceramic zirconia ferrules (Precision Fibre Products) were implanted in NAc shell (bregma coordinates: anterior/posterior, $1.3 \mathrm{~mm}$; medial/lateral, $1.6 \mathrm{~mm}$; dorsal/ventral, $-4.4 \mathrm{~mm} ; 10^{\circ}$ angle) and secured with dental cement (Grip Cement Dentsply). For robust viral expression in projection terminals of NAc shell, viral infusions occurred a minimum of 6 weeks before stimulation.

In vitro optogenetic patch-clamp electrophysiology. All recordings were conducted blind to the experimental conditions 2-28 days after CSDS. Whole-cell recordings were obtained from NAc MSNs in acute brain slices from mice that had been stereotaxically injected with AAV5-CaMKIIa-ChR2-EYFP into mPFC, vHIP or AMY. Eight to 12 weeks after viral surgery, mice were anaesthetized with isofluorane and perfused $1 \mathrm{~min}$ with ice-cold aCSF $(128 \mathrm{mM} \mathrm{NaCl}, 3 \mathrm{mM} \mathrm{KCl}$, $1.25 \mathrm{mM} \mathrm{NaH}_{2} \mathrm{PO}_{4}, 10 \mathrm{mM}$ D-glucose, $24 \mathrm{mM} \mathrm{NaHCO}, 2 \mathrm{mM} \mathrm{CaCl}_{2}$ and $2 \mathrm{mM}$ $\mathrm{MgCl}_{2}$; oxygenated with $95 \% \mathrm{O}_{2}$ and $5 \% \mathrm{CO}_{2}, \mathrm{pH} 7.4,295-305$ mOsm). Acute brain slices $(200 \mu \mathrm{m})$ containing NAc shell were cut using a microslicer (DTK1000 , Ted Pella) in cold sucrose aCSF ( $254 \mathrm{mM}$ sucrose, $3 \mathrm{mM} \mathrm{KCl}, 1.25 \mathrm{mM}$ $\mathrm{NaH}_{2} \mathrm{PO}_{4}, 10 \mathrm{mM}$ D-glucose, $24 \mathrm{mM} \mathrm{NaHCO}, 2 \mathrm{mM} \mathrm{CaCl} 2$ and $2 \mathrm{mM} \mathrm{MgCl}$; oxygenated with $95 \% \mathrm{O}_{2}$ and $\left.5 \% \mathrm{CO}_{2}, \mathrm{pH} 7.4,295-305 \mathrm{mOsm}\right)$. Slices were incubated in aCSF for $1 \mathrm{~h}$ at $32^{\circ} \mathrm{C}$ and then returned to room temperature for a minimum of $1 \mathrm{~h}$ before recording. Patch pipettes $(3-5 \mathrm{~m} \Omega$ ) for whole-cell voltageclamp recordings were filled with internal solution $(115 \mathrm{mM}$ potassium gluconate, $20 \mathrm{mM} \mathrm{KCl}, 1.5 \mathrm{mM} \mathrm{MgCl}_{2}, 10 \mathrm{mM}$ phosphocreatine, $10 \mathrm{mM}$ HEPES, $2 \mathrm{mM}$ magnesium ATP and 0.5 mM GTP; pH 7.2, $285 \mathrm{mOsm}$ ). Slices were continuously perfused with room temperature aCSF with $50 \mu \mathrm{m}$ picrotoxin (Sigma; flow rate $=2.5 \mathrm{ml} \mathrm{min}^{-1}$ ). Slices were examined to confirm robust viral expression at injection site and in NAc shell before proceeding. MSNs were voltage clamped at $-80 \mathrm{mV}$, and ChR2-expressing terminals were stimulated by blue light pulses generated by a stimulator (Agilent Technologies) and laser $(50 \mathrm{~mW}$; OEM laser systems) connected to a $200 \mu \mathrm{m}$ core optic fiber positioned above the slice aimed at the recorded cell. Optically evoked EPSCs were obtained every $20 \mathrm{~s}$ with paired pulses of $473 \mathrm{~nm}$ light ( $30 \mathrm{~mW}, 0.5-3 \mathrm{~ms} ; 100 \mathrm{~ms}$ interpulse interval) and recorded in voltage-clamp mode using the Multiclamp 700B amplifier. Data were acquired using pClamp 10 software (Molecular Devices). Series and access resistance were monitored during the experiments and signals were bessel filtered at $1.8 \mathrm{kHz}$ (gain10). Control experiments confirmed the stability of the PPR with the range of pulse widths $(0.5 \mathrm{~ms}-3 \mathrm{~s})$ and within a range of laser intensities $(10-30 \mathrm{~mW})$ although variability was observed at very low intensities $(5 \mathrm{~mW}$; Supplementary Fig. 7).

In vivo optogenetic electrophysiology. In vivo extracellular field recordings from NAc of anaesthetised intact mice were conducted following published methods. ${ }^{39}$ Mice were anaesthetised with $8 \%$ chloral hydrate $\left(400 \mathrm{mg} \mathrm{kg}^{-1}\right.$, i.p.). The skull was exposed and the area above the NAc was removed to lower an optrode consisting of a glass electrode (15-20 $\mathrm{M} \Omega$ filled with $2 \mathrm{mM} \mathrm{NaCl}$ ) tightly glued to an optic fiber. NAc coordinates were measured from bregma and were as follows (in $\mathrm{mm}$ ): anteroposterior, $1.7-0.86 \mathrm{~mm}$; mediolateral, $0-1.6 \mathrm{~mm}$; and dorsoventral, 3.3-4.7 mm. Using a DP-311 Differential Amplifier (Warner Instruments), extracellular optically evoked field responses were amplified $(\times 1,000)$ and filtered (0.3-1 kHz band pass). Optically evoked voltage data were acquired using an Axon Digidata Data Acquisition System (Molecular Devices), sampled using 16-bit resolution at $32 \mathrm{kHz}$, and stored using pCLAMP for further offline analysis.

In vivo optogenetic stimulation. Custom optical fiber patch cords attached to implanted fibers ${ }^{46}$ with ceramic zirconia sleeves (Precision Fiber Products) were connected using an FC/PC adaptor to a 473-nm blue laser diode (OEM Laser Systems; $100 \mathrm{~mW}$ ) and a stimulator to generate blue light pulses. LFS to induce LTD consisted of $10 \mathrm{~min}, 1 \mathrm{~Hz}, 4 \mathrm{~ms}$ pulse width $15-20 \mathrm{~mW}$, $473 \mathrm{~nm}$ stimulation $45 \mathrm{~min}$ before social interaction testing. In separate experiments, synaptic transmission was acutely increased during behavioural testing using $4 \mathrm{~Hz}, 5 \mathrm{~ms}$ pulse width, $15-20 \mathrm{~mW} 473 \mathrm{~nm}$ stimulation. This stimulation protocol has previously been shown to reliably modulate synaptic transmission by ChR2-expressing glutamatergic terminals in NAc ${ }^{12}$. Comparison of EYFP-injected stimulated controls and ChR2 injected nonstimulated controls showed no differences in social interaction behaviour (Supplementary Fig. 5).

CSDS and social interaction. All experiments utilized an established CSDS protocol to induce depressive-like behaviours in mice ${ }^{2}$. C57BL/6 J mice were subjected to 10 daily, 5-min defeats by a novel CD1 aggressor mouse and were then housed across a plexiglass divider to allow for sensory contact. Control mice were housed in cages separated from other control mice by a plexiglass divider and were rotated to a different cage daily. Resilient and susceptible mice were identified by their respective preference for, or avoidance of, interaction with a novel mouse after 10 days of defeat in a social interaction test. In vivo optogenetic experiments used the entire population of defeated mice and did not specifically select for resilient or susceptible to facilitate the detection of bidirectional shifts in social interaction behaviour.

Social-avoidance behaviour was assessed with a novel CD1 mouse in a twostage social-interaction test. In the first 2.5 -min test (no target), the experimental mouse was allowed to freely explore an arena $(44 \times 44 \mathrm{~cm})$ containing a plexiglass and wire mesh enclosure $(10 \times 6 \mathrm{~cm})$ against one wall of the arena. In the second 2.5-min test (target), the experimental mouse was returned to the arena with a novel CD1 mouse enclosed in the plexiglass wire mesh cage. Time spent in the 'interaction zone' $(14 \times 26 \mathrm{~cm})$ surrounding the plexiglass wire mesh cage, 'corner zones' $(10 \times 10 \mathrm{~cm})$ and 'distance travelled' within the arena was measured by video tracking software (Ethovision 5.0, Noldus).

Open field. Exploration of an open field arena $(44 \times 44 \mathrm{~cm})$ was assessed during a 5-min test. A video-tracking system (Ethovision 5.0, Noldus) measured locomotor activity, as well as the time spent in the centre $(34 \times 34 \mathrm{~cm})$ and periphery of the test arena as an index of anxiety.

Forced swim test. Mice were individually placed in beakers of $25^{\circ} \mathrm{C}$ water for 5 mins. Immobility was assessed by a video-tracking system (Ethovision 5.0, Noldus). We utilized a single-forced swim paradigm as extensively used and validated in mice ${ }^{3}$.

Cellular imaging. One hundred micrometres vibratomed coronal sections containing vHIP, mPFC or AMY, as well as respective NAc, from AAV infected mice, were mounted and coverslipped with ProLong Gold (Invitrogen). Sections were imaged for native EYFP fluorescence (GFP filter) on an Olympus BX51 upright immunofluorescence microscope. Images of viral injection sites (vHIP, mPFC and AMY) were imaged at $4 \times$ objective, and images of afferent axons in NAc were imaged at $10 \times$ objective. All images were obtained at equal gain and exposure and were further processed with NIH Image J. For quantification of immunohistochemistry slides were imaged on a Axio Observer Inverted Microscope (Zeiss) fitted with fluorescent filters. For each animal, sections along the A-P axis of the region of interest were imaged at the indicated magnification (AxioVision imaging software, Zeiss) by an observer blind to condition. Analysis of cell counts and colocalization was performed using ImageJ. Intensity thresholds were adjusted to accurately count moderate- to highly-expressing EGR1 + cells. Density is reported for cell counts to account for variation in region size. Statistical analysis was performed on the average from each animal when multiple sections were imaged and counted. For colocalization analysis, images were acquired on an inverted TCS SP8 laser scanning confocal microscope (Leica DMI 6000) and deconvolved (AutoQuant). Cells were imaged using a $60 \times$ oil immersion lens (numerical aperture 1.4; Leica) and a zoom of 1.0. Images were acquired with a resolution of $512 \times 512($ gain $=20 \%)$, and the line average was set to 4 . 
Statistics. No statistical methods were used to predetermine sample sizes but sample sizes are consistent with those reported in previous publications from our laboratory and sufficient for the types of experiments. Variance was similar between groups being statistically compared and data were normally distributed supporting the use of parametric statistics. Some animals or data points were lost in the course of social defeat, due to cage-flooding post-surgery, loss of fiber implant or equipment failure during behavioural testing. Grubb's test was used to identify and remove significant outliers from qPCR data. qPCR and paired-pulse data were analysed by one-way ANOVA and social interaction data were analysed by twoway repeated measures ANOVA. In all instances, Bonferroni post-hoc tests were used to resolve significant main effects or interactions. In one-way designs all pairwise comparisons were tested and in two-way repeated measures designs the effect of the between subjects variable was compared at each level of the within subjects variable. Two group comparisons (open field and forced swim test) were analysed by unpaired two-tailed Student's $t$-tests. Significance was set at $P<0.05$ for all tests and only comparisons with $P<0.05$ are explicitly reported. Detailed statistics are provided in figure legends. All data expressed as mean \pm s.e.m.

\section{References}

1. Krishnan, V. et al. Molecular adaptations underlying susceptibility and resistance to social defeat in brain reward regions. Cell 131, 391-404 (2007).

2. Berton, O. et al. Essential role of BDNF in the mesolimbic dopamine pathway in social defeat stress. Science 311, 864-868 (2006).

3. Vialou, V. et al. DeltaFosB in brain reward circuits mediates resilience to stress and antidepressant responses. Nat. Neurosci. 13, 745-752 (2010).

4. Christoffel, D. J. et al. IkappaB kinase regulates social defeat stress-induced synaptic and behavioral plasticity. J. Neurosci. 31, 314-321 (2011).

5. Lim, B. K., Huang, K. W., Grueter, B. A., Rothwell, P. E. \& Malenka, R. C. Anhedonia requires MC4R-mediated synaptic adaptations in nucleus accumbens. Nature 487, 183-189 (2012).

6. Epstein, J. et al. Lack of ventral striatal response to positive stimuli in depressed versus normal subjects. Am. J. Psychiatry. 163, 1784-1790 (2006).

7. Schlaepfer, T. E. et al. Deep brain stimulation to reward circuitry alleviates anhedonia in refractory major depression. Neuropsychopharmacology 33, 368-377 (2008)

8. Sesack, S. R. \& Grace, A. A. Cortico-basal ganglia reward network: microcircuitry. Neuropsychopharmacology 35, 27-47 (2010).

9. Goto, Y. \& Grace, A. A. Limbic and cortical information processing in the nucleus accumbens. Trends Neurosci. 31, 552-558 (2008).

10. French, S. J. \& Totterdell, S. Hippocampal and prefrontal cortical inputs monosynaptically converge with individual projection neurons of the nucleus accumbens. J. Comp. Neurol. 446, 151-165 (2002).

11. French, S. J. \& Totterdell, S. Individual nucleus accumbens-projection neurons receive both basolateral amygdala and ventral subicular afferents in rats. Neuroscience 119, 19-31 (2003).

12. Britt, J. P. et al. Synaptic and behavioral profile of multiple glutamatergic inputs to the nucleus accumbens. Neuron 76, 790-803 (2012).

13. MacAskill, A. F., Little, J. P., Cassel, J. M. \& Carter, A. G. Subcellular connectivity underlies pathway-specific signaling in the nucleus accumbens. Nat. Neurosci. 15, 1624-1626 (2012).

14. Goto, Y. \& Grace, A. A. Dopaminergic modulation of limbic and cortical drive of nucleus accumbens in goal-directed behavior. Nat. Neurosci. 8, 805-812 (2005).

15. Mulder, A. B., Hodenpijl, M. G. \& Lopes da Silva, F. H. Electrophysiology of the hippocampal and amygdaloid projections to the nucleus accumbens of the rat: convergence, segregation, and interaction of inputs. J. Neurosci. 18, 5095-5102 (1998).

16. Covington, 3rd H. E. et al. Antidepressant effect of optogenetic stimulation of the medial prefrontal cortex. J. Neurosci. 30, 16082-16090 (2010).

17. Vialou, V. et al. Prefrontal Cortical Circuit for Depression- and AnxietyRelated Behaviors Mediated by Cholecystokinin: Role of DeltaFosB. J. Neurosci. 34, 3878-3887 (2014).

18. Ressler, K. J. \& Mayberg, H. S. Targeting abnormal neural circuits in mood and anxiety disorders: from the laboratory to the clinic. Nat. Neurosci. 10, 1116-1124 (2007).

19. Chang, C. H. \& Grace, A. A. Amygdala-ventral pallidum pathway decreases dopamine activity after chronic mild stress in rats. Biol. Psychiatry. 76, 223-230 (2014).

20. Gill, K. M. \& Grace, A. A. Differential effects of acute and repeated stress on hippocampus and amygdala inputs to the nucleus accumbens shell. Int. J. Neuropsychopharmacol. 16, 2013-2025 (2013).

21. Gill, K. M. \& Grace, A. A. Heterogeneous processing of amygdala and hippocampal inputs in the rostral and caudal subregions of the nucleus accumbens. Int. J. Neuropsychopharmacol. 14, 1301-1314 (2011).

22. Floresco, S. B., Blaha, C. D., Yang, C. R. \& Phillips, A. G. Modulation of hippocampal and amygdalar-evoked activity of nucleus accumbens neurons by dopamine: cellular mechanisms of input selection. J. Neurosci. 21, 2851-2860 (2001).
23. Silver, R. A., Momiyama, A. \& Cull-Candy, S. G. Locus of frequencydependent depression identified with multiple-probability fluctuation analysis at rat climbing fibre-Purkinje cell synapses. J. Physiol. 510(Pt 3): 881-902 (1998).

24. Pascoli, V., Turiault, M. \& Luscher, C. Reversal of cocaine-evoked synaptic potentiation resets drug-induced adaptive behaviour. Nature 481, 71-75 (2012).

25. Pascoli, V. et al. Contrasting forms of cocaine-evoked plasticity control components of relapse. Nature 509, 459-464 (2014).

26. MacAskill, A. F., Cassel, J. M. \& Carter, A. G. Cocaine exposure reorganizes cell type- and input-specific connectivity in the nucleus accumbens. Nat. Neurosci. 17, 1198-1207 (2014).

27. Mayberg, H. S. et al. Regional metabolic effects of fluoxetine in major depression: serial changes and relationship to clinical response. Biol. Psychiatry. 48, 830-843 (2000).

28. Kennedy, S. H. et al. Changes in regional brain glucose metabolism measured with positron emission tomography after paroxetine treatment of major depression. Am. J. Psychiatry. 158, 899-905 (2001).

29. Mayberg, H. S. et al. Deep brain stimulation for treatment-resistant depression. Neuron 45, 651-660 (2005).

30. Hamani, C. et al. Antidepressant-like effects of medial prefrontal cortex deep brain stimulation in rats. Biol. Psychiatry. 67, 117-124 (2010).

31. Lobo, M. K. et al. DeltaFosB induction in striatal medium spiny neuron subtypes in response to chronic pharmacological, emotional, and optogenetic stimuli. J. Neurosci. 33, 18381-18395 (2013).

32. Francis, T. C. et al. Nucleus Accumbens Medium Spiny Neuron Subtypes Mediate Depression-Related Outcomes to Social Defeat Stress. Biol. Psychiatry. 77, 212-222 (2014)

33. Belujon, P. \& Grace, A. A. Restoring mood balance in depression: ketamine reverses deficit in dopamine-dependent synaptic plasticity. Biol. Psychiatry. 76, 927-936 (2014).

34. Holland, L. L. \& Wagner, J. J. Primed facilitation of homosynaptic long-term depression and depotentiation in rat hippocampus. J. Neurosci. 18, 887-894 (1998).

35. van Beugen, B. J., Qiao, X., Simmons, D. H., De Zeeuw, C. I. \& Hansel, C. Enhanced AMPA receptor function promotes cerebellar long-term depression rather than potentiation. Learn. Mem. 21, 662-667 (2014).

36. Siddoway, B., Hou, H. \& Xia, H. Molecular mechanisms of homeostatic synaptic downscaling. Neuropharmacology 78, 38-44 (2014).

37. Mannie, Z. N. et al. Structural and functional imaging of the hippocampus in young people at familial risk of depression. Psychol. Med. 44, 2939-2948 (2014).

38. Jaworska, N., Yang, X. R., Knott, V. \& Macqueen, G. A review of fMRI studies during visual emotive processing in major depressive disorder. World J. Biol. Psychiatry. doi: 10.3109/15622975.2014.885659 (17 March 2014).

39. Cao, J. L. et al. Mesolimbic dopamine neurons in the brain reward circuit mediate susceptibility to social defeat and antidepressant action. J. Neurosci. 30, 16453-16458 (2010).

40. Chaudhury, D. et al. Rapid regulation of depression-related behaviours by control of midbrain dopamine neurons. Nature 493, 532-536 (2013).

41. Floresco, S. B., Todd, C. L. \& Grace, A. A. Glutamatergic afferents from the hippocampus to the nucleus accumbens regulate activity of ventral tegmental area dopamine neurons. J. Neurosci. 21, 4915-4922 (2001).

42. Maze, I. et al. Essential role of the histone methyltransferase G9a in cocaineinduced plasticity. Science 327, 213-216 (2010).

43. Basu, K. et al. Novel strategy to selectively label excitatory and inhibitory neurons in the cerebral cortex of mice. J. Neurosci. Methods 170, 212-219 (2008).

44. Felix-Ortiz, A. C. \& Tye, K. M. Amygdala inputs to the ventral hippocampus bidirectionally modulate social behavior. J. Neurosci. 34, 586-595 (2014).

45. Stuber, G. D. et al. Excitatory transmission from the amygdala to nucleus accumbens facilitates reward seeking. Nature 475, 377-380 (2011).

46. Sparta, D. R. et al. Construction of implantable optical fibers for long-term optogenetic manipulation of neural circuits. Nat. Protoc. 7, 12-23 (2012).

\section{Acknowledgements}

This work was supported by the National Institute of Mental Health (R01MH051399 and P50MH096890 to E.J.N. and R01MH092306 to M.H.H.), the Hope for Depression Research Foundation, Johnson \& Johnson/International Mental Health Research Organization Rising Star Translational Research Award (M.H.H.), and National Natural Science Foundation of China (81200862 to H.X.Z).

\section{Author contributions}

R.C.B. and E.J.N. designed the research; R.C.B., E.M.P, C.J.P, H.X.Z, I.M., D.C., B.P., performed the research; R.C.B. and C.J.P., analysed the data; R.C., C.A.B-G., J.F.C., K.D 
and M.H.H. provided reagents and technical training necessary to perform the research; R.C.B. \& E.J.N. wrote the paper.

\section{Additional information}

Supplementary Information accompanies this paper at http://www.nature.com/ naturecommunications

Competing financial interests: The authors declare no competing financial interests.

Reprints and permission information is available online at http://npg.nature.com/ reprintsandpermissions/
How to cite this article: Bagot, R.C. et al. Ventral Hippocampal Afferents to the Nucleus Accumbens Regulate Susceptibility to Depression. Nat. Commun. 6:7062 doi: 10.1038/ ncomms8062 (2015).

(c) (i) This work is licensed under a Creative Commons Attribution 4.0 International License. The images or other third party material in this article are included in the article's Creative Commons license, unless indicated otherwise in the credit line; if the material is not included under the Creative Commons license, users will need to obtain permission from the license holder to reproduce the material. To view a copy of this license, visit http://creativecommons.org/licenses/by/4.0/ 


\section{Corrigendum: Ventral hippocampal afferents to the nucleus accumbens regulate susceptibility to depression}

Rosemary C. Bagot, Eric M. Parise, Catherine J. Peña, Hong-Xing Zhang, lan Maze, Dipesh Chaudhury, Brianna Persaud, Roger Cachope, Carlos A. Bolaños-Guzmán, Joseph F. Cheer, Karl Deisseroth, Ming-Hu Han \& Eric J. Nestler

Nature Communications 6:7062 doi: 10.1038/ncomms8062 (2015); Published 8 May 2015; Updated 17 Jun 2015

In the original version of this Article, the middle initial of the author Joseph F. Cheer was omitted from the author information. The affiliation details for this author were also incorrect. These errors have now been corrected in both the PDF and HTML versions of the article. 